\title{
An improved non-equilibrium model for the ignition of living fuel
}

\author{
A. Lamorlette $^{\mathrm{A}, \mathrm{C}}$, M. El Houssami ${ }^{\mathrm{B}}$ and D. Morvan ${ }^{\mathrm{A}}$

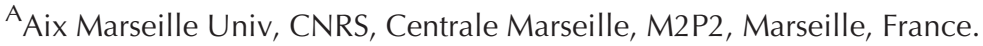 \\ ${ }^{B}$ BRE Centre for Fire Safety Engineering, The University of Edinburgh, UK. \\ ${ }^{\mathrm{C}}$ Corresponding author. Email: aymeric.lamorlette@univ-amu.fr
}

\begin{abstract}
This paper deals with the modelling of living fuel ignition, suggesting that an accurate description using a multiphase formulation requires consideration of a thermal disequilibrium within the vegetation particle, between the solid (wood) and the liquid (sap). A simple model at particle scale is studied to evaluate the flux distribution between phases in order to split the net flux on the particles into the two sub-phases. An analytical solution for the split function is obtained from this model and is implemented in ForestFireFOAM, a computational fluid dynamics (CFD) solver dedicated to vegetation fire simulations, based on FireFOAM. Using this multiphase formulation, simulations are run and compared with existing data on living fuel flammability. The following aspects were considered: fuel surface temperature, ignition, flaming combustion time, mean and peak heat release rate (HRR). Acceptable results were obtained, suggesting that the thermal equilibrium might not be an acceptable assumption to properly model ignition of living fuel.
\end{abstract}

\section{Introduction}

Detailed studies on ignition and combustion of wildland fuel led to the development of multiphase formulations to precisely describe the behaviour of fuels during fire propagation (Grishin 1997; Larini et al. 1998; Morvan and Dupuy 2001; SéroGuillaume and Margerit 2002; Mell et al. 2009). These models include a full description of fuel degradation, along with a lowMach number formulation to represent the flow behaviour. They have demonstrated their ability to properly describe fire propagation on dead fuel (Grishin 1997; Larini et al. 1998; Morvan and Dupuy 2001; Mell et al. 2009) and their ability to accurately predict heat release rate (HRR) of dead fuel at medium scale (Mell et al. 2009), ignition time, mass loss and HRR at small scale (El Houssami et al. 2016). However, these models are unable to properly predict behaviour of living fuel (Pickett et al. 2010). Indeed, as pointed out by Pickett et al. (2010) and McAllister et al. (2012), large temperature gradients are observed within fuel particles, with a plateau at $\sim 200-300^{\circ} \mathrm{C}$ for the particle surface temperature and a plateau at $\sim 140^{\circ} \mathrm{C}$ for the core temperature, during evaporation (Pickett et al. 2010). This behaviour, specific to living fuel, cannot be accounted for using a classical multiphase formulation as in Grishin (1997), Larini et al. (1998), Morvan and Dupuy (2001), Séro-Guillaume and Margerit (2002), Mell et al. (2009) and El Houssami et al. (2016), because the thermal equilibrium assumption (between the solid and the liquid comprising the fuel particles) and the classical degradation models lead to a plateau at $100^{\circ} \mathrm{C}$ for the particle temperature. Moreover, the work of Mell et al. (2009) does not show comparison between experimental results and numerical simulations for a fuel moisture content (FMC) value higher than $49 \%$, suggesting that this model requires further investigation for high FMC. Finally, as discussed in El Houssami (2016), the classical multiphase formulation can properly predict ignition time, mass loss and HRR of forest fuel such as pitch pine needles in the configuration of the FM Global Fire Propagation Apparatus (FPA), as long as the initial FMC is lower than $50 \%$; however, it fails to properly predict behaviour for higher initial FMC. For FMC higher than $100 \%$, error on ignition time becomes greater than $100 \%$, error on flaming combustion time becomes greater than $50 \%$ and errors on peak and mean HRR become greater than $60 \%$; Such huge errors highlight that the classical multiphase model cannot predict flammability properties of living fuels, which often exhibit FMC greater than $100 \%$, at least in the FPA configuration. That is the reason why the multiphase formulation has to be adapted in order to handle high initial FMC, as observed in the case of living fuel. In a recent article by Anand et al. (2017), the authors tackled the problem of living fuel by separately considering free and bound water. Bound water was introduced to the multiphase model, for which thermokinetic evaporation parameters are considered to be higher than for free water, leading to a higher value of the solid phase temperature during the evaporation of this part of water contained in vegetation. In this study, we propose to adapt the multiphase formulation for living fuel, suggesting a disequilibrium between the solid and the liquid phases within the fuel particles. This assumption is suggested for the following reasons:

- Solid particles can exhibit temperature above $100^{\circ} \mathrm{C}$, up to $200-300^{\circ} \mathrm{C}$ while still containing water. 

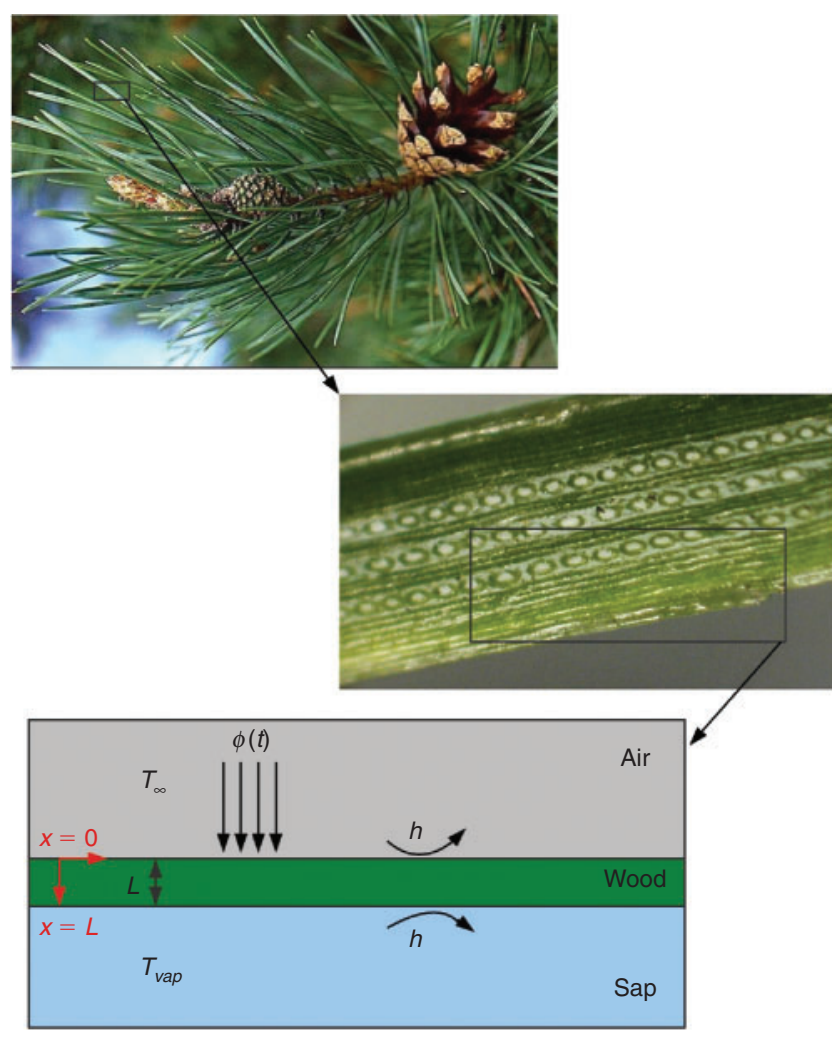

Fig. 1. Sketch of the problem.

- The large amount of free water in living fuel, contained in the fuel particle core, is not dispersed in the particles and is rather isolated (Simpson and TenWolde 1999), suggesting that these two phases could be out of equilibrium.

- According to Pickett et al. (2010), the $140^{\circ} \mathrm{C}$ plateau inside the particles could correspond to sap evaporation. Therefore the particles show an inner temperature approximately equal to evaporation temperature, while the surface temperature is much higher. This observation suggests that sap could be approximated as water, with a slightly higher evaporation temperature.

In order to evaluate this disequilibrium, we study a simplified living particle ignition configuration, at particle scale, as shown in Fig. 1. This configuration considers a solid film over a liquid at constant (evaporation) temperature. The solid film is heated by radiation and cooled by convection on the front side, whereas on the rear side exchange is a convective flux with the liquid under it. The problem is then reduced to a one-dimensional finite-depth heat diffusion problem, as suggested in the classical ignition theory presented in Torero (2008), applied to vegetation in Torero and Simeoni (2010) and adapted to finite depth solids (Lamorlette and Candelier 2015), in order to evaluate the amount of heat actually reaching the liquid phase during the heating process leading to ignition. The present study provides insight on the flux distribution between phases.

The mathematical formulation is then implemented in ForestFireFOAM (El Houssami et al. 2016) and simulations are run to compare particle temperature before ignition with the results of Pickett et al. (2010). Indeed, classical multiphase models usually provide solid temperature evolution with a $100^{\circ} \mathrm{C}$ plateau during evaporation. Therefore, temperature estimations from the disequilibrium model will be qualitatively analysed. Then, as an attempt to validate the model, numerical predictions of ignition time and HRR will be compared to experimental results using pitch pine needle bed Thomas (2016). These needles exhibit very high fuel moisture content, suggesting that they are still alive. This choice has been made based on the results of El Houssami (2016), which show that only experiments conducted with an initial FMC higher than $50 \%$ would be relevant to validate the model presented here, since the classical equilibrium model has been proved to be legitimate for lower initial FMC. Also, these experimental results have been chosen based on our previous studies. Indeed, as shown in El Houssami (2016) and El Houssami et al. (2016), the FPA configuration simulations using ForestFireFOAM can be considered as validated on dead pitch pine needle bed, hence experiments in the FPA with living pitch pine needles seem the best candidate to validate the model presented here. In our study, prediction of ignition time, flaming time and HRR time evolution are compared with a set of two experiments on live fuel with an initial FMC equal to $110 \%$. Additionally, results are compared with experimental correlations developed in Thomas (2016) on ignition time and peak HRR as a function of initial FMC in the range $70-150 \%$.

This present paper is organised as follows: the 'Finite-depth conduction problem' section is dedicated to the one-dimensional finite-depth heat diffusion problem where an analytical solution is provided. An expression for the ratio of the heat reaching the liquid phase over the heat reaching the solid during the ignition process is obtained. The 'Implementing the flux ratio in a multiphase formulation' section describes the implementation of the previous solution in a multiphase formulation. Modification of the classical multiphase formulation are presented below. The 'Comparison between experiments and numerical simulations' section presents a numerical study and its comparison with experimental results on the following aspects: particle outer temperature plateau, ignition time, flaming time, HRR, peak HRR and average HRR during flaming combustion. Unfortunately, mass loss and temperature were not measured in this configuration. Nevertheless, as shown in El Houssami et al. (2016), mass loss and HRR in multiphase simulations are rather correlated, hence HRR and ignition time comparisons seem sufficient to evaluate, at least partially, the proposed approach. Indeed, ignition time is representative of the fuel behaviour during the local heating and evaporating phases leading to ignition so it is an acceptable indicator of the fuel behaviour regarding its moisture content, before ignition. Then HRR gives an acceptable insight on the fuel behaviour after ignition, according to the correlation observed in El Houssami et al. (2016) between HRR and mass loss. Finally, conclusions on the thermal disequilibrium are drawn based on the previous comparison between experimental results and numerical simulations.

\section{Finite-depth conduction problem}

In order to evaluate the flux distribution between the natural polymers comprising the solid phase (referred to as 'wood' in 
the rest of the article) and sap within a vegetation particle, the problem is modelled in its simpler form, that is, without considering any topological effect due to the particle geometry. Hence, the conduction problem is solved in Cartesian coordinates, using a simple model for the solid film. Therefore the following assumptions are suggested: the film shrinkage that could occur during the heating phase before ignition is neglected, the solid contact with evaporating sap is modelled using a mixed boundary condition and the char layer that could form just before ignition occurs is considered small enough to always be at steady-state so that heat transfer within this layer does not influence the model. These assumptions will be discussed in this article. Furthermore, it is proposed that water vapour will be able to flow out of the particles through small cracks, without changing the pressure inside the particles (hence the evaporation temperature) nor affecting the temperature profile in the solid. This aspect is supported by observations of Pickett et al. (2010) about the $140^{\circ} \mathrm{C}$ plateau inside the particles. In contrast, the work of McAllister et al. (2012) suggests otherwise, that large pressure gradients are responsible for cracks in particles. In our study, the authors consider that these pressure gradients do not last in time and occur only during the crack formation. Hence, when the vapour leaves the particles, the process occurs at constant pressure that is in accordance with a temperature plateau inside the particles, as observed by Pickett et al. (2010). Both phases are considered at equilibrium before evaporation begins, so that the initial temperature of the solid phase is considered homogeneous and equal to the evaporation temperature. This approach follows the methodology developed within the frame of the classical ignition theory presented in Torero (2008), applied to vegetation in Torero and Simeoni (2010) and adapted to finite depth solid in Lamorlette and Candelier (2015). The same assumptions and mixed radiative boundary conditions are chosen. Indeed, the solution accuracy of the classical ignition theory on ignition time prediction (see, for example, Torero 2008) suggests that such a model, with its underlying assumptions and boundary conditions, is representative of a realistic ignition setup. Hence any in-depth radiation effect in the solid film is also neglected. The assumptions of the classical ignition theory are detailed below (for a thorough discussion on these assumption relevancy, see Torero 2008):

- the solid is considered as being inert until ignition,

- ignition occurs when the surface solid temperature reaches ignition temperature,

- radiant heating (without in-depth effects) and convective cooling are considered on the exposed (front) face of the solid,

- convective cooling is considered at the rear face of the solid, and

- constant, time-averaged parameters are considered (conductivity, density, specific heat and total heat transfer coefficient).

\section{Solving the conduction problem}

A one-dimensional inert solid material of depth $L$ is considered with one side exposed to an incoming heat flux $\phi(t)$, as represented in Fig. 1. The heat conduction equation is now expressed for the solid target, introducing:

$$
\theta(x, t)=\left(T(x, t)-T_{\infty}\right) / T_{\infty}
$$

where $T(x, t)$ is the solid temperature, $T_{\infty}$ is the temperature of the surrounding air, $T_{\text {vap }}$ is the evaporation temperature and the initial temperature so that $\theta_{\text {vap }}=\left(T_{\text {vap }}-T_{\infty}\right) / T_{\infty}$ and $T_{i g}$ is the solid (wood) ignition temperature; $\lambda$ is the solid (wood) heat conductivity, $\rho_{w}$ is its density, $C_{p_{w}}$ is its specific heat and $h$ is a total heat transfer coefficient. The following quantities are suggested to normalise equations:

$$
\begin{gathered}
\mathrm{Bi}=\frac{h L}{\lambda} \\
\Phi=\frac{\phi L}{\lambda T_{\infty}} \\
t^{*}=\frac{t \lambda}{\rho_{w} C_{p_{w}} L^{2}} ; x^{*}=\frac{x}{L}
\end{gathered}
$$

The dimensionless heat diffusion problem thus reads (superscript asterisks are removed for clarity):

$$
\frac{\partial \theta(x, t)}{\partial t}=\frac{\partial^{2} \theta(x, t)}{\partial x^{2}}
$$

with the following boundary conditions:

$$
\begin{gathered}
-\frac{\partial \theta(x, t)}{\partial x}=\Phi-\operatorname{Bi} \theta(x, t), x=0 \\
-\frac{\partial \theta(x, t)}{\partial x}=\operatorname{Bi}\left[\theta(x, t)-\theta_{\text {vap }}\right], x=1 \\
\theta(x, 0)=\theta_{\text {vap }}, \forall x
\end{gathered}
$$

Eqns 5 and 6 are similar to the boundary conditions used in the classical ignition theory (Torero 2008 and Lamorlette and Candelier 2015), except that temperature is set to evaporation instead of ambient temperature to mimic the contact between the solid and evaporating water, at $x=1$. A mixed boundary condition is suggested at $x=1$ because boiling sap under heavy temperature gradients (as observed in Pickett et al. 2010) could be responsible for a fluid motion under the solid film. Eqn 5 can be considered realistic for an igniting particle configuration, such as the one presented in Fig. 1 (according to the classical ignition theory). However Eqn 6 is more questionable, because a Dirichlet boundary condition can also be applied in this configuration. For mathematical simplicity, the mixed boundary condition that has been suggested is kept, as it does not influence the effective flux distribution between wood and sap. This aspect is discussed in the next section, after deriving the flux distribution function.

In order to get a general solution for an arbitrary function $\phi(t)$, let us first consider the response of Eqn 4 to a sudden constant heat flux $\phi(t)=\phi H(t)$, where $H(t)$ is the Heaviside step function. The classical following methodology is now applied to solve these equations, according to Liukov (1968): a steadystate solution $\theta_{0}$ is first sought for Eqns 4 to 6 , then a timedependent perturbation $\theta_{1}$ is sought using separation of 
variables, with $\theta=\Phi \cdot\left(\theta_{0}+\theta_{1}\right)$ due to Eqn 4 linearity. The problem admit the following solution:

$$
\theta_{1}=e^{-X^{2} t}\left(C_{1} \cos X x+C_{2} \sin X x\right)
$$

Introducing $\theta_{1}$ in Eqns 5 and 6 provides the trivial solution $C_{1}=C_{2}=0$ because the problem relative to the temperature perturbation is homogeneous. Hence, the system determinant is set to zero to obtain the only non-zero solution possible. Thus Eqns 5 and 6 lead to the following characteristic equation, according to Liukov (1968):

$$
\tan X_{n}=\frac{2 \mathrm{Bi} X_{n}}{X_{n}^{2}-\mathrm{Bi}^{2}}
$$

This equation is not solved in Liukov (1968). Indeed, root determination for this equation requires numerical means. However in the present article, the first root determination provides a solution that is acceptable, as described later. $\theta_{1}$ becomes:

$$
\theta_{1}=\sum_{n=1}^{\infty} C_{n} e^{-X_{n}^{2} t}\left(\cos X_{n} x+\frac{\mathrm{Bi}}{X_{n}} \sin X_{n} x\right)
$$

with $\Phi\left[\theta_{1}(t=0)+\theta_{0}\right]=\theta_{\text {vap }}$, leading to:

$$
\begin{aligned}
\sum_{n=1}^{\infty} & C_{n}\left(\cos X_{n} x+\frac{\mathrm{Bi}}{X_{n}} \sin X_{n} x\right) \\
= & \frac{\theta_{\text {vap }}}{\Phi}-\left[\frac{\mathrm{Bi} \theta_{\text {vap }} / \Phi-1}{(\mathrm{Bi}+2)} x+\frac{1+\mathrm{Bi}\left(1+\theta_{\text {vap }} / \Phi\right)}{\mathrm{Bi}(\mathrm{Bi}+2)}\right]
\end{aligned}
$$

Orthogonality of the following function $h\left(X_{n} x\right)$ has been proved in Lamorlette and Candelier (2015), hence coefficients $C_{n}$ can be calculated:

$$
h\left(X_{n} x\right)=\cos X_{n} x+\frac{\mathrm{Bi}}{X_{n}} \sin X_{n} x
$$

Solution $\theta$ is then constructed solving numerically Eqn 8 . This solution is, however, complex to handle because of the infinite sum. Nevertheless, a careful study of this range of application shows that ignition is always occurring at $t_{i g}>0.5$ for an ignition temperature $T_{i g}=600 \mathrm{~K}$. This observation is based on typical values of $\mathrm{Bi}$ and $\Phi$ for particles such as pine needles, leaves or twigs in the context of fire ignition and propagation, as in Lamorlette and Candelier (2015) and Lamorlette (2014). Making a comparison of the solution computed with the first hundred roots of Eqn 8 with a one-mode solution proves that these two solutions are matching at $t=0.1$. Hence, the first term of the sum accurately describes behaviour of vegetation particles during ignition, as long as the first root of Eqn 8 is properly estimated. Here the value $X_{1}=\sqrt{2 \mathrm{Bi}}-\sqrt{2} \mathrm{Bi}^{3 / 2} / 12$ is chosen, as obtained in Lamorlette and Candelier (2015).

\section{Evaluating the flux distribution between the solid and the fluid phases}

Now that the conduction problem is solved, its solution is investigated evaluating the flux received by the living fuel particle before its ignition. The flux is split between wood and sap resulting in separate solutions for the wood temperature equation and for the sap temperature equation in this new multiphase formulation. Indeed, if a disequilibrium exists between wood and sap, the solid phase equation of the classical multiphase formulation cannot be used anymore and two different equations have to be solved for wood and sap temperature. This requires evaluating the net heat flux fractions reaching wood and sap. According to the sketch presented in Fig. 1, the part of the net heat flux that actually reaches the sap to turn it into vapour before ignition is equal to:

$$
T_{\infty} \int_{0}^{t_{i g}} \lambda \frac{\partial \theta}{\partial x_{x=1}} d t
$$

whereas the net heat flux on the solid film is equal to:

$$
T_{\infty} \int_{0}^{t_{i g}} \lambda \frac{\partial \theta}{\partial x_{x=0}} d t
$$

Hence the ratio $R_{\Phi}$ of the net surface heat flux reaching the liquid on the net surface heat flux hitting the solid film during the ignition process, which can be presented as a split function, can be expressed as:

$$
R_{\Phi}=\frac{\int_{0}^{t_{i g}} \frac{\partial \theta}{\partial x_{x=1}} d t}{\int_{0}^{t_{i g}} \frac{\partial \theta}{\partial x_{x=0}} d t}
$$

Because only the first root of Eqn 8 has to be taken into account, $t_{i g}$ can be calculated analytically and inserted into $R_{\Phi}$, such that $R_{\Phi}$ has a fully explicit expression. This expression is not provided in this article for clarity and also because it is practically hazardous to implement because of its length. Nevertheless, an accurate approximation can be obtained considering a third order series expansion in $\mathrm{Bi}$ and a cut-off at the critical heat flux corresponding to the steady-state solution $\theta_{0}$. Hence, one gets:

$$
\begin{aligned}
R_{\Phi}= & \frac{349 \Phi^{2}-180 \theta_{\text {vap }}^{2}-420 \Phi\left(\theta_{i g}-\theta_{\text {vap }}\right)-720 \theta_{\text {vap }} \theta_{i g}+900 \theta_{i g}^{2}}{2160 \Phi^{2}} \mathrm{Bi}^{2} \\
& +\frac{-12619 \Phi^{3}-15120 \Phi \theta_{\text {vap }}^{2}-22680 \theta_{\text {vap }}^{3}+23142 \Phi^{2}\left(\theta_{i g}-\theta_{\text {vap }}\right)}{181440 \Phi^{3}} \mathrm{Bi}^{3} \\
& +\frac{65520 \Phi \theta_{\text {vap }} \theta_{i g}+37800 \theta_{\text {vap }}^{2} \theta_{i g}-50400 \Phi \theta_{i g}^{2}-98280 \theta_{v a p} \theta_{i g}^{2}+83160 \theta_{i g}^{3}}{181440 \Phi^{3}} \mathrm{Bi}^{3} \\
& +\frac{-2 \Phi+3\left(\theta_{i g}-\theta_{\text {vap }}\right)}{6 \Phi} \mathrm{Bi}, \Phi>\Phi_{c r}, \\
& R_{\Phi}=1, \Phi \leq \Phi_{c r}
\end{aligned}
$$

where $\Phi_{c r}=\left[\theta_{i g}(\mathrm{Bi}+2)-\theta_{\text {vap }}\right] \mathrm{Bi} /(\mathrm{Bi}+1), \theta_{i g}=1.048$ and $\theta_{\text {vap }}=0.341$, corresponding to $T_{i g}=600 \mathrm{~K}, T_{\infty}=293 \mathrm{~K}$. This model is rather insensitive to $T_{\text {vap }}$ in the range $393-433 \mathrm{~K}$, hence it has been taken at $T_{v a p}=393 \mathrm{~K}$. Indeed, according to Eqn 15, $R_{\Phi}$ is not sensitive to a realistic range of $\theta_{\text {vap }}$. Hence, the expression of the split function is little influenced by the rear face boundary condition (Eqn 6), for small $\mathrm{Bi}$ in particular. Therefore $R_{\Phi}$ would show a very similar trend if a Dirichlet boundary condition had been used instead of Eqn 6 . 


\section{Implementing the flux ratio in a multiphase formulation}

Now that the distribution function has been evaluated, a new multiphase formulation considering thermal disequilibrium inside the vegetation particles is proposed. The full set of equations is not presented here. Readers can refer to section 2 of Morvan et al. (2009) for the general formulation (solid phase equations, gas phase transport equations and radiation equations) and to El Houssami et al. (2016) for the sub-models (solid phase degradation, solid-gas phase interactions, turbulence and combustion). Only equations that are modified are presented in this study.

A volumetric definition of the FMC $\left(F M C_{v}\right)$ is suggested in this study, opposed to the classical fuel moisture content $(F M C)$ definition that considers a mass ratio. Hence,

$$
F M C=F M C_{v} \frac{\rho_{l}}{\rho_{w}}
$$

where $\rho_{l}$ is the liquid (water) density. It allows to define the total volumetric fraction of solid $\left(\alpha_{s}\right)$ as the sum of the wood volumetric fraction $\left(\alpha_{w}\right)$ plus the liquid volumetric fraction $\left(\alpha_{l}\right)$ the following way:

$$
\alpha_{s}=\alpha_{w}+\alpha_{l}=\alpha_{w}\left(1+F M C_{v}\right)
$$

Hence, the mass of liquid per unit volume can be defined as $\alpha_{w} \rho_{l} F M C_{v}$. This definition is suggested to remove the wood density from the liquid phase equations (FMC and temperature). As $\rho_{w}$ evolves due to pyrolysis and char production, it could create numerical issues in the liquid phase equations. This is avoided using the $F M C_{v}$ definition. The following consideration is also taken into account to properly implement the flux distribution function: because it is assumed that the char layer that could form over the solid film just before ignition is small enough to always be at steady-state, the radiant heat flux on this layer is recovered just underneath the char, which is at the top of the solid film. Hence, the positive flux $\phi$ that should be considered at the top of the solid film in the definition of $\Phi$ is composed of this radiant heat flux, in addition to the char combustion contribution to the solid phase that is produced in the char layer.

The first equation to modify because of thermal disequilibrium is naturally the wood temperature equation, which becomes:

$$
\alpha_{w} \rho_{w} C_{p_{w}} \frac{d T_{w}}{d t}=\left(1-R_{\Phi}\right)\left(Q_{c o n v}+Q_{r a d}-\alpha_{c h a r} \Delta h_{c h a r} W_{c h a r}\right)-\Delta h_{p y r} W_{p y r}
$$

where $T_{w}$ is the wood temperature, $Q_{c o n v}$ is the convective heat flux, $Q_{\text {rad }}$ is the net radiant heat flux, $\alpha_{c h a r}$ is the fraction of the charring energy released in the solid phase, $\Delta h_{\text {char }}$ is the charring combustion enthalpy, $\Delta h_{p y r}$ is the pyrolysis reaction enthalpy, $W_{c h a r}$ is the charring reaction rate and $W_{p y r}$ is the pyrolysis reaction rate.

An equation for the liquid is now added to the system:

$\alpha_{w} \rho_{l} F M C_{v} C_{p l} \frac{d T_{l}}{d t}=R_{\Phi}\left(Q_{c o n v}+Q_{\text {rad }}-\alpha_{c h a r} \Delta h_{\text {char }} W_{\text {char }}\right)-\Delta h_{\text {vap }} W_{\text {vap }}$ where $C_{p_{l}}$ is the specific heat of the liquid, $T_{l}$ is the liquid temperature, $\Delta h_{\text {vap }}$ is the evaporation enthalpy and $W_{\text {vap }}$ is the evaporation reaction rate. Note that summing Eqns 18 and 19 and considering equilibrium (i.e. $T_{w}=T_{l}$ ) leads to the solid temperature equation that is used in classical multiphase formulations. The new formulation allows the liquid temperature to be equal to the solid temperature until evaporation begins, then it allows the liquid temperature to remain at evaporating temperature during the whole evaporation phase, while the solid temperature is free to increase.

The new $F M C$ equation now reads:

$$
\frac{d\left(\alpha_{w} \rho_{l} F M C_{v}\right)}{d t}=-W_{\text {vap }}
$$

with the evaporation reaction rate defined as:

$$
\begin{gathered}
W_{\text {vap }}=0, T_{l}<T_{\text {vap }} \\
W_{\text {vap }}=R_{\Phi}\left(Q_{\text {conv }}+Q_{\text {rad }}-\alpha_{\text {char }} \Delta h_{\text {char }} W_{\text {char }}\right) / \Delta h_{\text {vap }}, T_{l} \geq T_{\text {vap }}
\end{gathered}
$$

Now, according to the analytical development that led to $R_{\Phi}$ expression, the thermal equilibrium between wood and sap has to be forced for $T_{w}<T_{v a p}$ to fit the initial condition of the analytical problem. Hence, $R_{\Phi}$ is driven by Eqn 15 only if $T_{w}<T_{\text {vap }}$. In the case where $T_{w}<T_{\text {vap }}$, the equilibrium has to be recovered, which corresponds to $T_{w}=T_{l}$. Introducing this constraint in Eqns 18 and 19 leads to the following value for $R_{\Phi}$ :

$$
R_{\Phi}=\frac{F M C_{v} \rho_{l} C_{p l}}{\rho_{w} C_{p_{w}}+F M C_{v} \rho_{l} C_{p l}}, T_{w}<T_{v a p}
$$

The equation that solved the volumetric solid fraction now solves the wood volumetric fraction. Hence, this equation does not change but aims to solve for $\alpha_{w}$ instead of $\alpha_{s}$ :

$$
\rho_{w} \frac{d \alpha_{w}}{d t}=-W_{c h a r}
$$

The total volumetric fraction can then be calculated after solving $\alpha_{w}$ and $F M C_{v}$, using Eqn 17. Finally the evaporation term $W_{\text {vap }}$ is suppressed from the wood mass equation since it was added to the liquid mass equation ( $F M C_{v}$ equation).

In practice, Bi and $\Phi$ are calculated at every iteration, for each cell containing the solid phase, based on the local values and calculated fluxes that are directly accessible in the code. Then, in order to solve Eqns 18 and $19, R_{\Phi}$ is calculated in each cell containing the solid phase. It is noted here that the value of the total heat transfer coefficient is almost constant until $R_{\Phi}$ reaches its quasi-steady value of 1 , which is consistent with the approach used to derive its expression.

It is important to highlight that the specific area ratio gives a good approximation of the particle thickness; however, it does not allow any estimation of the film thickness $L$. As a first approximation, a cylindrical model is proposed (as for needles) and the film thickness is estimated as half the particle diameter $L=2 / \sigma_{s}$, where $\sigma_{s}$ is the vegetation particle specific area ratio. 
A parameter $k_{\text {film }}$ is then added into the model to study the behaviour of the system for different effective film thickness. Hence the effective film thickness $L$ is defined as:

$$
L=2 k_{f i l m} / \sigma_{s}
$$

This parameter is first varied in this study to check its sensitivity.

\section{Comparison between experiments and numerical simulations}

Multiphase models such as Grishin (1997) have been designed considering an averaging over a vegetation-representative elementary volume; however, experiments of Pickett et al. (2010) have been conducted at particle scale, which is smaller than what can be represented using the multiphase approach. Hence, we chose to simulate ignition at an intermediate scale, i.e. the FM Global FPA scale, as in El Houssami et al. (2016) and Thomas (2016). The experiments that are used as a comparison with the numerical simulations (on ignition time and HRR) were done on living pitch pine needles. The experiments are extracted from Thomas (2016). The experimental set-up is briefly described in a specific sub-section. These simulations are expected to provide similar, homothetic dynamics at slower evolution rates, due to the change of scale (which is smaller in the experiments of Pickett et al. 2010) and imposed heat fluxes (which are higher in the experiments of Pickett et al. 2010). Indeed, even though the heat fluxes are different in Pickett et al. (2010) (i.e. mainly convective), the particle temperature behaviour is expected to be the same because it mainly depends on the net incoming heat flux and the heat conduction phenomena within the particles. Hence, the results of Pickett et al. (2010) are only used for a qualitative comparison on temperature trends in order to check if the proposed formulation allows to shift the temperature plateau value observed during evaporation.

\section{Sub-models and parameters}

Sub-models and parameters specific to the simulations presented in this study are listed below:

- Momentum interaction is modelled using a classical Forchheimer term, with a drag coefficient $C_{d}=0.1$, suited for the needle bed considered in this study, according to Nepf (1999) and El Houssami (2016), for a solid fraction of $8.2 \%$ and cylindrical particles.

- Convective heat transfer is modelled through a natural convection model (Irvine and Hartnett 1978):

$$
\mathrm{Nu}=h D / \lambda=C(\mathrm{Gr} \operatorname{Pr})^{n}
$$

with $D$ being the particle diameter (linked to the specific area ratio), Gr is the Grashof number, Pr is the Prandtl number, $C=0.119$ and $n=0.3$ are model constants (El Houssami et al. 2016).

- The pyrolysis model is a two-step Arrhenius model (Morvan and Dupuy 2001; Mell et al. 2009):

$$
W_{p y r}=\alpha_{s} \rho_{w} Y_{d r y} K_{p y r} e^{\frac{-E_{p y r}}{R T_{w}}}
$$

where $T_{w}<800 \mathrm{~K}$.
Table 1. Properties used in the study

\begin{tabular}{lc}
\hline$\rho_{w}\left(\mathrm{~kg} \mathrm{~m}^{-3}\right)$ & 831.7 \\
$C_{p_{w}}\left(\mathrm{~J} \mathrm{~kg}^{-1} \mathrm{~K}^{-1}\right)$ & 2069 \\
$\lambda\left(\mathrm{W} \mathrm{m}^{-1} \mathrm{~K}^{-1}\right)$ & 0.14 \\
$\Delta h_{\text {char }}\left(\mathrm{J} \mathrm{kg}^{-1}\right)$ & $-3 \times 10^{7}$ \\
$\Delta h_{\text {pyr }}\left(\mathrm{J} \mathrm{kg}^{-1}\right)$ & $4.18 \times 10^{5}$ \\
$K_{\text {pyr }}\left(\mathrm{s}^{-1}\right)$ & 3640 \\
$K_{\text {char }}\left(\mathrm{m} \mathrm{s}^{-1}\right)$ & 430 \\
$E_{\text {pyr }} / R(\mathrm{~K})$ & 7250 \\
$E_{\text {char }} / R(\mathrm{~K})$ & 8000 \\
\hline \multicolumn{2}{c}{} \\
$\left(Q_{\text {conv }}+Q_{\text {rad }}-\alpha_{\text {char }} \Delta h_{\text {char }} W_{\text {char }}\right) / \Delta h_{\text {pyr }}$
\end{tabular}

where $T_{w} \geq 800 \mathrm{~K}$ and $Y_{d r y}$ is the dry wood mass fraction, $K_{p y r}$ is the pre-exponential factor and $E_{p y r}$ is the activation energy. The first step corresponds to the model of Di Blasi et al. (2001), successfully used in El Houssami et al. (2016) to model pyrolysis. It has been adapted to the present study to address the previous remark on the char combustion contribution to the net surface heat flux. The pyrolysis reaction transforms dry wood into carbon monoxide and char.

- The char combustion model is a simple Arrhenius model (Morvan and Dupuy 2001):

$$
W_{\text {char }}=\frac{\alpha_{S} \sigma_{s}}{v_{o_{2}}} \alpha_{g} \rho Y_{o_{2}} K_{c h a r} e^{\frac{-E_{c h a r}}{R T_{w}}}
$$

where $\alpha_{g}$ is the porosity, $Y_{\mathrm{O}_{2}}$ is the oxygen mass fraction, $K_{\text {char }}$ is the pre-exponential factor and $E_{\text {char }}$ is the activation energy.

- The turbulence and combustion models are based on a oneequation model for the turbulent kinetic energy (Shaw and Patton 2003), with an additional sink term due to dissipation of sub-grid scale energy (Morvan et al. 2009). A modified eddy dissipation concept (EDC) model (Ren et al. 2016) is used to account for the laminar behaviour of the flame at its basis. The chemical reaction is simplified to a one step reaction converting carbon monoxide into carbon dioxide.

- Finally, a correction for the mean absorption of radiation by vegetation in the frequency range of the FPA heaters has to be accounted for, as suggested in Chaos (2014) and El Houssami et al. (2016). The correction is here slightly different, according to El Houssami (2016), because the needles are fresh. Hence, the correction on the FPA heaters heat flux is $\alpha_{\text {eff }}=0.72$ and the heat flux imposed by the FPA heaters in the simulation is $F_{\text {sim }}=\alpha_{e f f} F_{x p}$, where $F_{x p}$ is the heat flux imposed by the FPA heaters in the experiments, as in El Houssami et al. (2016). Please note that the FPA heaters heat flux $F$ is different from the heat flux $\phi$ that reaches the particles, as, for the particles on the top of the bed, $F$ is just a portion of $\phi$ once ignition occurred as the flame is then responsible for an additional radiant heat flux on the fuel bed.

Parameters that are used in the numerical simulations are summarised in Table 1 (El Houssami 2016; El Houssami et al. 2016).

\section{Experimental setup}

First, two sets of experimental results performed by Thomas (2016) with the FM Global FPA are used for the present study. 


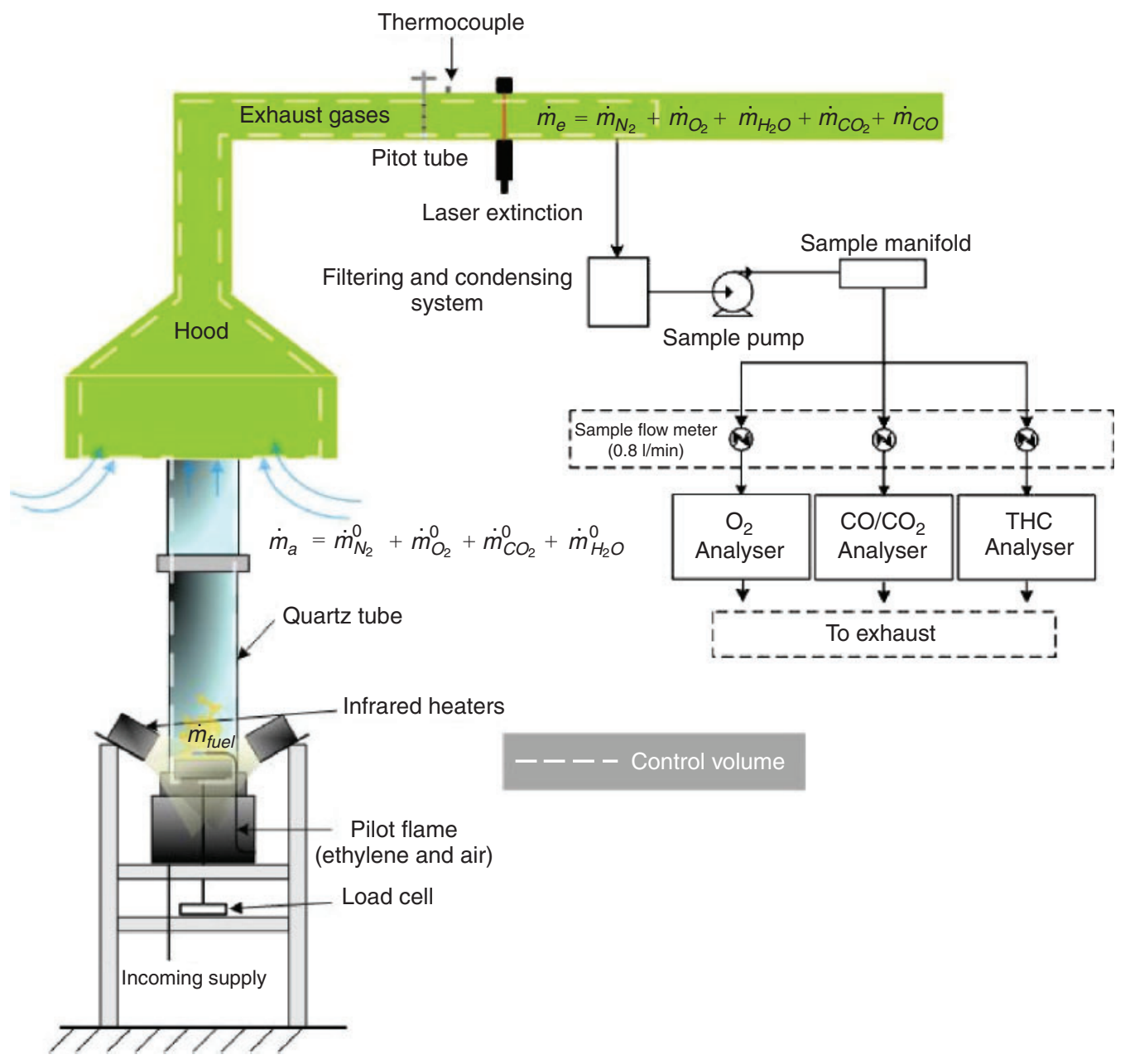

Fig. 2. Sketch of the factory mutual (FM) Global Fire Propagation Apparatus (FPA) configuration.

The FPA (Fig. 2) provides controlled and repeatable conditions (Schemel et al. 2008; El Houssami et al. 2016), such as the ability to produce a constant incident radiative heat flux. No forced flow was applied in our study, only natural convection was allowed through the porous samples. The quartz tube has been removed, hence, sample boundaries are open to the atmosphere, with an ambient temperature at $293 \mathrm{~K}$. Live pitch pine needles were packed in cylindrical open baskets of $12.6-\mathrm{cm}$ diameter and $3-\mathrm{cm}$ depth. The vegetation sample parameters are as follows: $\alpha_{s}=8.2 \%, \sigma_{s}=7112 \mathrm{~m}^{-1}, F M C=110 \%$. In these experiments, HRR was measured using calorimetry (Schemel et al. 2008). Ignition time and flaming combustion time are also recorded.

Second, another set of experiments is considered in the present study, using pitch pine needles on a broad FMC range, which has been conducted and used in Thomas (2016) to develop correlations on ignition time and peak HRR as a function of FMC.

\section{Numerical setup}

The set of transport equations in the gas phase are solved using a second order implicit finite volume method. Total variation diminishing (TVD) schemes have been adopted to avoid introduction of false numerical diffusion (Patankar 1980). The set of ordinary differential equations governing the evolution of the solid fuel was solved using a second order explicit method. A constant time step $\Delta t=0.002 \mathrm{~s}$ is used to achieve temporal accuracy and numerical stability. This has been checked running a sensitivity study on the time step. This value leads to a mean Courant-Friedrichs-Lewy (CFL) at $\sim 0.4$, where CFL is defined as:

$$
\mathrm{CFL}=u \Delta t / \Delta x
$$

where $u$ is the local velocity in a cell and $\Delta x$ is the mesh resolution in the same cell. In the present study, only twodimensional calculations are performed. The overall domain is a rectangle of $1 \mathrm{~m}$ wide and $1.5 \mathrm{~m}$ high. A simple overview of the numerical domain and the sensitivity study are presented here because the numerical conditions are very similar to the study of El Houssami (2016) and El Houssami et al. (2016). Hence, refer to El Houssami (2016) for more details, where the numerical representation of the FPA configuration has been thoroughly investigated. The mesh was composed primarily of hexahedral 
cells. Based on a mesh sensitivity study, a mesh resolution of $(0.001 \mathrm{~m})^{2}$ was determined as providing converging results in the vicinity of the sample and near the lamps. The mesh is stretched above the lamps and beyond the zone of interest until the boundaries in order to reduce computational time. The side boundaries are open, allowing entrainment of air. However, the ground has a wall boundary condition applied for velocity and a Dirichlet condition for temperature. An outlet boundary condition is set at the top above the flame, representing the exhaust hood. The temperature of the entrained air from the open boundaries is at $293 \mathrm{~K}$. The sample is $3 \mathrm{~cm}$ deep as in the FPA configuration. Lamps were modelled by fixing a constant heat flux on specific walls. These have the same view factor as in the FPA, giving the same heat flux experimentally on the sample surface $\left(25 \mathrm{~kW} \mathrm{~m}^{-2}\right)$. A first simulation is made with the following vegetation sample parameters: $\alpha_{s}=8.2 \%$, $\sigma_{s}=7112 \mathrm{~m}^{-1}, F M C=110 \%$. Four other simulations are run with FMC equal to $70,90,130$ and $150 \%$, where the dry mass has been kept equal to the first simulation in order to match the experimental conditions of Thomas (2016).

Simulations are run for the following range of $k_{\text {film }}: k_{\text {film }}$ $\in 0.25-1.5$. Next section illustrates the results for the different $k_{f i l m}$ values and a comparison with experimental data.

\section{Result comparison}

\section{Qualitative comparison with Pickett's data}

Even if, as stated earlier, experiments of Pickett et al. (2010) cannot be numerically reproduced using a multiphase formulation, a qualitative comparison is first done on the solid surface temperature with the numerical results obtained in the FPA configuration. This comparison only aims to show that the suggested model will not lead to a $100^{\circ} \mathrm{C}$ plateau for the solid phase during evaporation, which is now known to be wrong, according to Pickett et al. (2010).

Results of the numerical simulations are presented in Fig. 3 for different $k_{\text {film }}$ values and also for the 'equilibrium' model in the FPA configuration. Fig. 4 shows a curve extracted from Pickett et al. (2010) for manzanita leaf (an arbitrary choice because no data for a species close to pitch pine were available directly in Pickett et al. 2010) as a comparison. It is first noticed that the model is hardly sensitive to $k_{\text {film }}$; nevertheless, the value

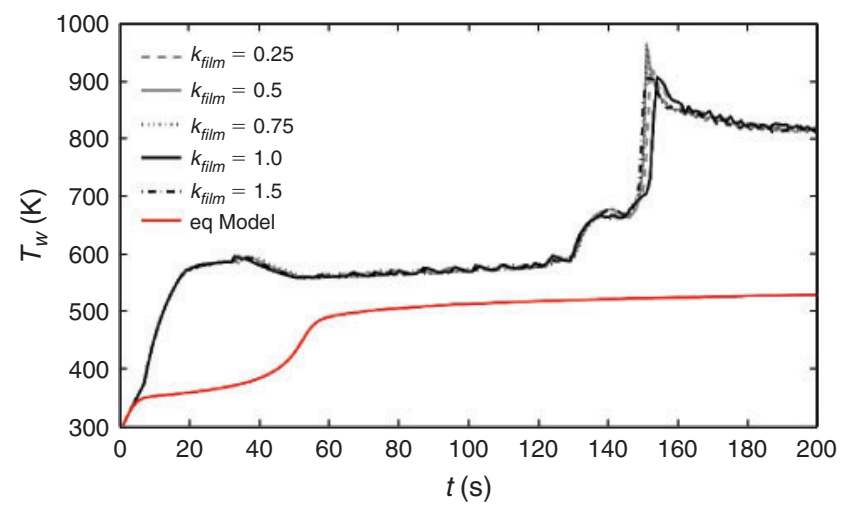

Fig. 3. Surface solid temperature for different $k_{\text {film }}$ values and for the equilibrium model, for a $25 \mathrm{~kW} \mathrm{~m}^{-2}$ radiant heat flux. of $k_{\text {film }}=0.25$ is maintained for the rest of the article as it provides the best HRR curve in comparison with the results of Thomas (2016) for the same needle bed and configuration. This aspect will be discussed later. The model is shown to be insensitive to the parameter $k_{\text {film }}$ in the range studied here, hence, the film thickness does not influence the global behaviour of the bed. This suggests that the assumption of a negligible shrinkage of the solid film during the drying phase is acceptable.

Contrary to the former 'equilibrium' model, the surface temperature evolution predicted by the new model describes the same trend and dynamic as in the experimental results of Pickett et al. (2010) but slower, as expected because of the sample size, as shown in Figs 3 and 4. First, it exhibits a prompt temperature increase, followed by a dampening and an overshot. Then the temperature stabilises at the temperature plateau described by Pickett et al. (2010), at $\sim 200-300^{\circ} \mathrm{C}$, during evaporation. The numerical simulation also describes a second and a third temperature raise at $t=130 \mathrm{~s}$ and $t=144 \mathrm{~s}$, corresponding to a local and global ignition of the fuel sample respectively. Here, ignition corresponds to thermal runaway. The third temperature rise is associated with char combustion on the upper layer of the sample, which is unlikely to occur at the scale of Pickett et al. (2010) experiments. This could explain why the temperature evolution is different at large time scale. Hence, this result highlights the major difference in the solid phase behaviour between the equilibrium model and the new approach.

Again, the considerable differences between the configuration of Pickett et al. (2010) and the FPA configuration do not allow a proper validation. Nevertheless, these results demonstrate that a non-equilibrium model allows evaporation at a higher (and more realistic) temperature plateau than predicted using the previous multiphase model.

\section{Quantitative comparison with Thomas data at} $F M C=110 \%$

A quantitative comparison is now performed in the FPA configuration, between experiments conducted by Thomas (2016) and numerical simulations for an initial FMC of $110 \%$ evaluating ignition time and HRR time evolution.

In the simulation of the FPA configuration, local ignition occurs at $t=130 \mathrm{~s}$, which corresponds to the experimental data

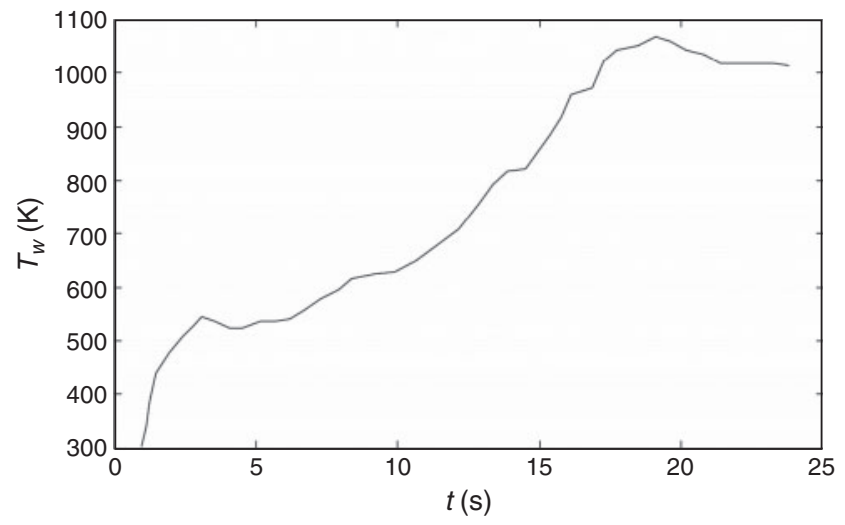

Fig. 4. Data extracted from Pickett et al. (2010) for manzanita leaf. 
of Thomas (2016) (experimental ignition occurs at $t=132 \mathrm{~s}$, hence the error is $1.5 \%$ ). If global ignition was to be considered, it occurs in the simulation at $t=144 \mathrm{~s}$, which would lead to a $9.5 \%$ error. In comparison, the equilibrium model shows an ignition time greater than $250 \mathrm{~s}$. The result of the disequilibrium model is acceptable, contrary to the result of the equilibrium model, suggesting that the disequilibrium approach proposed in the present study is relevant to describe the temperature evolution process leading to ignition.

The HRR evolution is presented in Fig. 5 with a time offset to compensate for the error on the ignition time, showing an acceptable agreement with the two corresponding experiments of Thomas (2016), presented using two different symbols in Fig. 5. Flaming combustion time is computed at $86 \mathrm{~s}$, against $116 \mathrm{~s}$ in the experiment, leading to a $26 \%$ error. According to El Houssami et al. (2016), the gas phase combustion model is mainly responsible for this underestimation. The peak HRR at

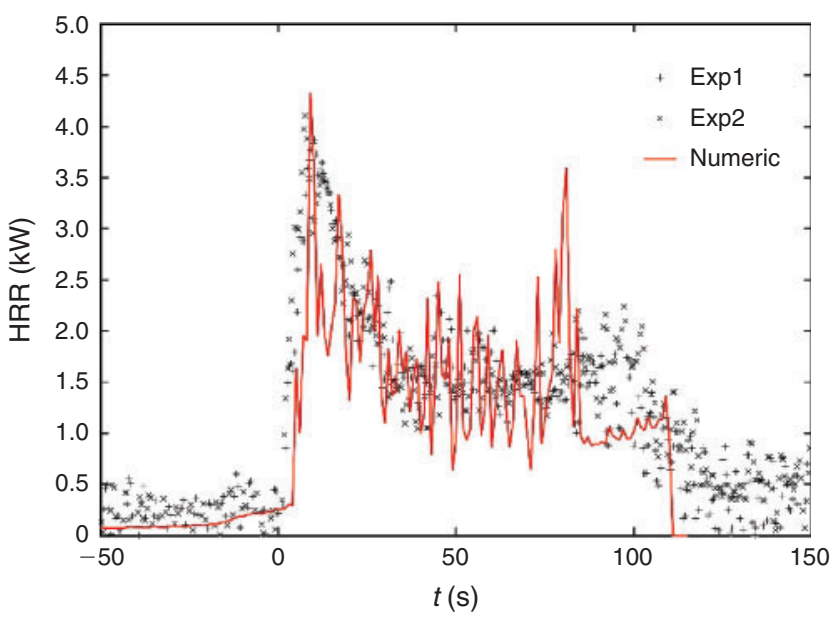

Fig. 5. Heat Release Rate with time offset for a $25 \mathrm{~kW} \mathrm{~m}^{-2}$ radiant heat flux. Experimental results are represented using symbols and numerical results are represented by the solid line. ignition is evaluated at $4.32 \mathrm{~kW}$ against $3.98 \mathrm{~kW}$ in the experiment, leading to an $8.6 \%$ error. The mean HRR during flaming combustion is $1.64 \mathrm{~kW}$ against $1.71 \mathrm{~kW}$ in the experiment, hence, the error is $4.2 \%$. For comparison, El Houssami (2016) showed that the equilibrium model predicted a flaming combustion time under $50 \mathrm{~s}$, with mean HRR and peak HRR at ignition more than three time lower than the experimental results. This confirms the need to discard the usual equilibrium assumption for living fuels. Finally, the peak HRR at flameout is $3.59 \mathrm{~kW}$ against $1.99 \mathrm{~kW}$ in the experiment; this is the largest error, equal to $81 \%$. This large error, which is the only trend that the model did not accurately predict, is also responsible for the flaming time misestimation. Moreover, it occurs once all the FMC has evaporated. It suggests that the pyrolysis control through a two-step Arrhenius is not sufficient to properly capture the behaviour at flameout.

Quantitative comparison with the correlations developed by Thomas (2016) for FMC in the range 70-150\%

A comparison is now performed between the numerical results obtained for FMC in the range $70-150 \%$ and the correlations developed by Thomas (2016) on ignition time and peak HRR as a function of FMC.

In Thomas (2016), the following correlation was obtained for pitch pine needle bed ignition time in the FMC range $0-150 \%$ under a $25 \mathrm{~kW} \mathrm{~m}^{-1}$ radiant heat flux:

$$
t_{i g}=40.938+0.9255 \frac{F M C}{100}
$$

The results obtained with the full set of numerical simulations is plotted against Eqn 28 in Fig. 6. As for the 110\% FMC case, local and global ignitions are plotted, showing an acceptable agreement if local ignition is considered and a good agreement if global ignition is considered. Indeed, the mean error on local ignition is $8.8 \%$ and the mean error on global ignition is $2.2 \%$. Surprisingly, contrary to the $110 \%$ FMC case that has been investigated before, global ignition is closer to the

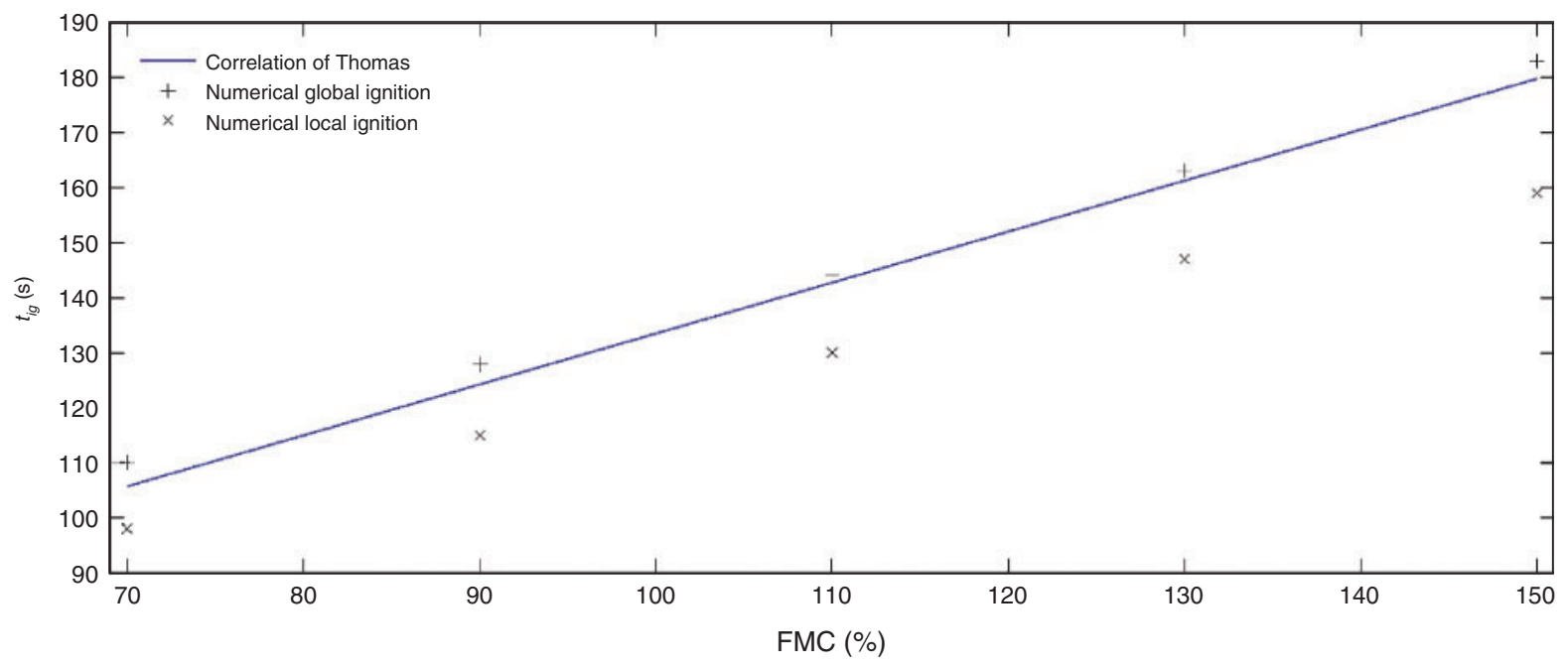

Fig. 6. Ignition time correlation of Thomas (2016) for a $25 \mathrm{~kW} \mathrm{~m}^{-2}$ radiant heat flux and numerical results obtained in this study. 


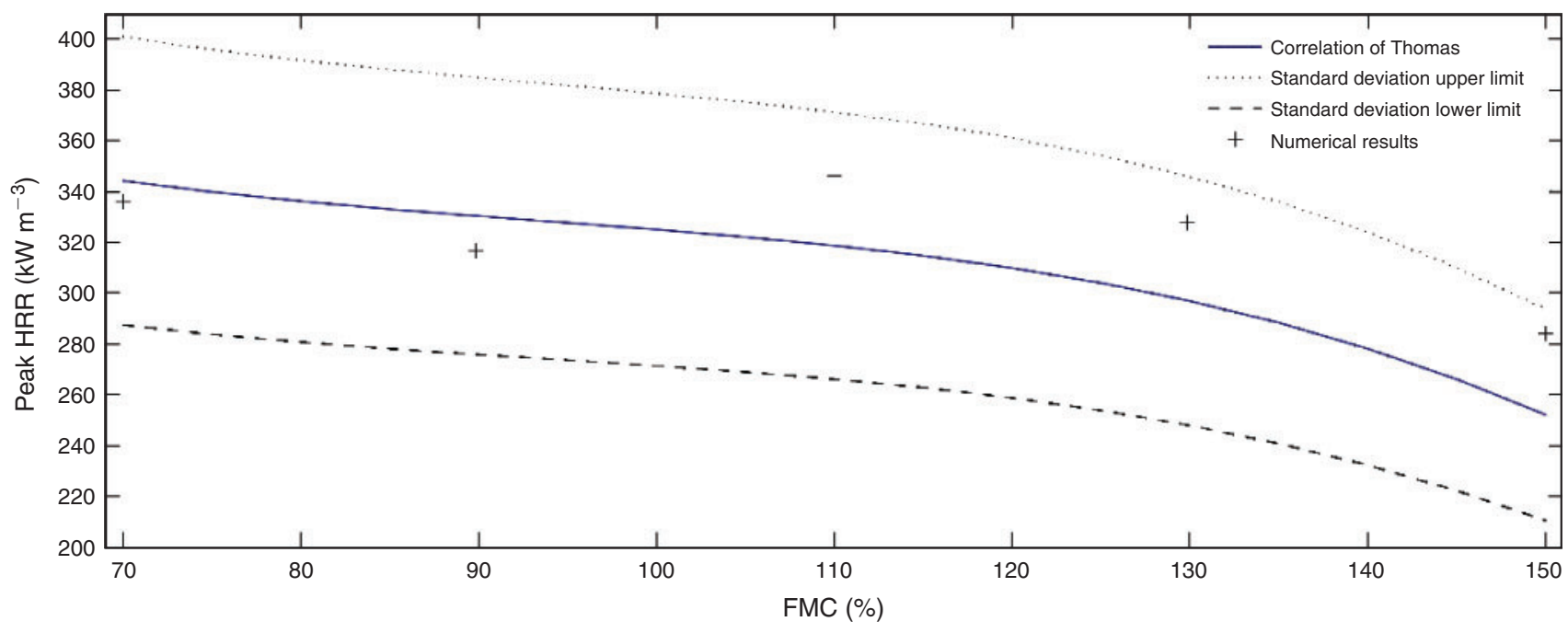

Fig. 7. Peak heat release rate (HRR) correlation of Thomas (2016) for a $25 \mathrm{~kW} \mathrm{~m}^{-2}$ radiant heat flux with standard deviation and numerical results obtained in this study.

correlation than local ignition. It should be first noted here that the correlation value at $110 \%$ is not strictly the experimental value of the ignition time, which could explain this behaviour. Also, this difference might be due to seasonal effects, as discussed in Thomas (2016), as this correlation has been established on more than 150 experiments, with needles sampled during different seasons. Thomas (2016) observed that small variations in ignition time and HRR can be due to these seasonal effects. Nevertheless, the error on ignition time remains acceptable.

In Thomas (2016), the following correlation was obtained for pitch pine needle bed peak HRR in the FMC range $0-150 \%$ under a $25 \mathrm{~kW} \mathrm{~m}^{-2}$ radiant heat flux, where the peak HRR is reduced by the surface of the sample:

$H R R_{\text {peak }}=594-263.37 F M C^{3}+740.52 F M C^{2}-746.13 F M C$

The results obtained with the full set of numerical simulations is plotted against Eqn 29 and its standard deviation in Fig. 7, shows an acceptable agreement. Indeed, the mean error is $7.5 \%$ and the maximum error is $12.6 \%$, which is lower than the $16.5 \%$ standard deviation of this correlation (Thomas 2016). Additionally, as observed and discussed in Thomas (2016), the peak HRR is weakly sensitive to the FMC value in the range $60-120 \%$, whereas a drop is observed for higher FMC values. This drop is not clear with the numerical results for the $130 \%$ FMC case, but it is clearly observed for a $150 \%$ FMC. Hence the global trend of the peak HRR dependency on FMC can be considered as acceptably reproduced.

These comparisons suggest that the new formulation presented in the present study can acceptably predict the flammability properties of living pitch pine needles on a broad FMC range for which the classical multiphase formulation has been proved to be inaccurate in the FPA configuration (El Houssami 2016). Hence, it suggests that this new formulation, which

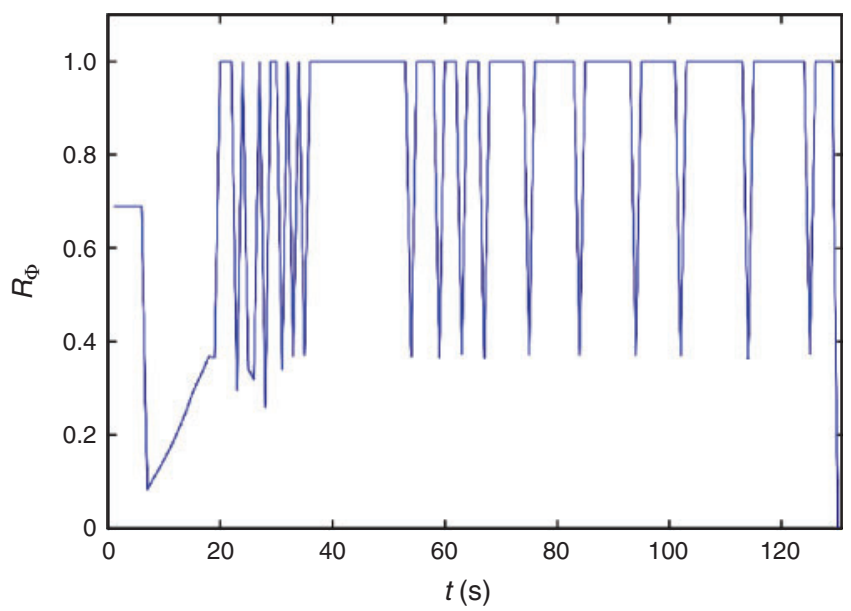

Fig. 8. $R_{\Phi}$ evolution at the sample surface before ignition.

considers thermal disequilibrium between wood and sap inside fuel particles, is appropriate to predict the behaviour of living fuel.

\section{Discussion}

Suggesting thermal disequilibrium between wood and free water within vegetation particle seems an appropriate strategy to model ignition of living fuel. Nevertheless, it is surprising to see that the simplest model of flux splitting between phases provides an accurate behaviour at sample scale, with very few sensitivity of the model to its parameter $k_{\text {film }}$. An analysis of the flux splitting during the numerical simulations can explain this behaviour. Indeed, according to Fig. 8, the behaviour of the splitting function is very stiff. Once evaporation temperature is reached (at $t=\sim 12 \mathrm{~s}$ ), the solid continues to heat taking the largest part of the net flux until it reaches an equilibrium 
between the surface fluxes (radiant, convective and charring) and the loss in the liquid phase (at $t=\sim 12 \mathrm{~s}$ ). When the solid film reaches this point, then $R_{\Phi}=1$ and the net heat flux is only responsible for evaporation. This equilibrium temperature corresponds to the $200-300^{\circ} \mathrm{C}$ plateau described by Pickett et al. (2010). These fluxes, however, cannot keep the solid film at equilibrium because this equilibrium temperature allows pyrolysis to begin. As a consequence, the pyrolysis reaction slowly cools the solid film and reduces radiant and convective losses until the net flux becomes greater than the critical heat flux. This behaviour is responsible for several sharp decreases of $R_{\Phi}$ that happen regularly during the evaporation process (as the behaviour of $R_{\Phi}$ is very stiff close to the critical heat flux). Every time this process occurs, a slight decrease followed by a slight increase of the solid temperature is observed, as shown in Fig. 3 between 20 and $130 \mathrm{~s}$. Every small temperature increase is associated with variation of $R_{\Phi}$ from 1 to a value $\sim 0.4$, as shown in Fig. 8. Consequently, $R_{\Phi}$ stabilises at 1 until the solid film temperature again reaches a value for which the net flux becomes greater than the critical heat flux and the process repeats itself until ignition, where $R_{\Phi}$ drops to 0 . This result suggests that further refinements of the analytical model are not necessary to properly model the disequilibrium as the splitting is expected to be mainly driven by the critical heat flux value, which mainly depends on ignition temperature, as pointed out in the 'Evaluating the flux distribution between the solid and the fluid phases' section. Hence, it seems that no topological effect or in-depth radiation effect need to be taken into account in the splitting function model. Nevertheless, it is observed that ignition occurs once pyrolysis and charring have begun, hence, the assumption of an inert solid until ignition seems to breaks down. This could suggest that a more detailed description of the solid is needed. However, these phenomena occur after $R_{\Phi}$ reached its quasi-steady value of 1 while a plateau is observed for the solid temperature. It means that the solid film and the char layer are in a quasi-steady-state during this phase, which fits the assumption made on the char layer behaviour, which is used to simplify the heat transfer model. It also means that the flux distribution between wood and sap is not sensitive to these phenomena (pyrolysis and charring) any longer as the whole heat diffusion problem reached a steady-state.

This result also shows how disequilibrium affects thermal degradation. Indeed, instead of having full evaporation at $100^{\circ} \mathrm{C}$ followed by a temperature raise and thermal degradation, this model led to a higher plateau for evaporation, which implies more surface losses during the evaporation process. However, it allows thermal degradation while particles are not yet fully dried; hence, it seems that evaporation can slow down the degradation process in driving the solid surface temperature evolution. This numerical behaviour, which seems in line with experimental observations, is the consequence of the introduction of the split function in the multiphase model.

Finally, it should be noted that, in the work of Anand et al. (2017), ignition time is acceptably predicted in the FIST apparatus (see McAllister et al. (2012) for a description of this apparatus) with both the classical multiphase formulation and the corrected formulation that take into account the bound water effect, using wildland-urban interface fire dynamics simulator (WFDS). This result indicates that the approach presented in the present article is not always necessary, depending on the experimental configuration: heat fluxes in Anand et al. (2017) are much greater than in our study, resulting to ignition times shorter $(30 \mathrm{~s})$ than those obtained in our study (100 s) for the same FMC range. The different heat fluxes lessen the effect of water evaporation dynamics on the global ignition dynamic, justifying why the classical multiphase formulation could be acceptable for living fuels in the experimental conditions such as presented in McAllister et al. (2012).

\section{Conclusion}

A thermal disequilibrium between solid and liquid phases inside vegetation particles seems mandatory in a multiphase model for live needles to properly reproduce experimental behaviours. Indeed, in the FPA configuration, it allows predicting experimental behaviour specific to living fuel, such as the $200-300^{\circ} \mathrm{C}$ temperature plateau on the vegetation particle surface, occurring during evaporation, which earlier models fail to capture. In addition, the model is successfully able to predict ignition time, flaming time, peak HRR at ignition and mean HRR during flaming combustion on a validation case and agrees with experimental correlations obtained on ignition time and peak HRR on a broad range of FMC with live needles. In contrast, the classical multiphase formulation failed to reproduce these results for high FMC values. However, the flameout is not properly reproduced in this study, suggesting that the end of the pyrolysis process (i.e. when the sample is fully dry) needs a correction to properly capture living fuel behaviour. Nevertheless, this model now needs to be tested against more experimental data in order to fully validate the approach and its range of applications, as pointed out in the Discussion section. This validation would first require measurement of fuel particle temperature in the bed before ignition and mass loss during the whole degradation and combustion phases. Second, experiments should be conducted on other vegetation species to investigate the effect of the solid properties such as the density and the specific heat, but also to test the split function for different values of $\mathrm{Bi}$, under different imposed heat flux values. Finally, comparison to experiments on propagation configurations should follow to complete the studies conducted on static configurations.

\section{Conflicts of interest}

The authors declare that they have no conflicts of interest.

\section{Nomenclature}

$C$, Nusselt correlation pre-exponential factor (unitless).

$C_{n}$, perturbation coefficient (unitless).

$C_{d}$, drag coefficient (unitless)

$C_{p}$, specific heat $\left(\mathrm{J} \mathrm{kg}^{-1} \mathrm{~K}^{-1}\right)$.

$\Delta h$, combustion enthalpy $\left(\mathrm{J} \mathrm{kg}^{-1}\right)$.

$\Delta t$, time step (s).

$\Delta x$, mesh resolution $(\mathrm{m})$.

$E / R$, activation temperature $(\mathrm{K})$.

$F$, heat flux imposed by the FPA heaters $\left(\mathrm{W} \mathrm{m}^{-2}\right)$.

$h$, total heat transfer coefficient $\left(\mathrm{W} \mathrm{m}^{-2} \mathrm{~K}^{-1}\right)$.

$\mathrm{HRR}$, heat release rate $(\mathrm{kW})$. 
$K_{\text {char }}$, charring pre-exponential factor $\left(\mathrm{m} \mathrm{s}^{-1}\right)$.

$K_{\text {pyr }}$, pyrolysis pre-exponential factor $\left(\mathrm{s}^{-1}\right)$.

$k_{\text {film }}$, film thickness parameter (unitless).

$L$, solid film depth (m).

$n$, Nusselt correlation exponent (unitless).

$Q$, volumetric heat flux $\left(\mathrm{W} \mathrm{m}^{-3}\right)$.

$R_{\Phi}$, flux distribution function (unitless).

$t$, time (s).

$T$, temperature $(\mathrm{K})$.

$u$, local velocity $\left(\mathrm{m} \mathrm{s}^{-1}\right)$.

$W$, reaction rate $\left(\mathrm{s}^{-1}\right)$.

$x$, position (m).

$X_{n}$, solution of the characteristic equation (unitless).

$Y_{d r y}$, dry wood mass fraction (unitless).

$Y_{\mathrm{O}_{2}}$, oxygen mass fraction (unitless).

\section{Greek symbols}

$\alpha$, volumetric fraction (unitless).

$\alpha_{c h a r}$, charring fraction (unitless).

$\alpha_{e f f}$, incident heat flux correction (unitless).

$\lambda$, heat conductivity $\left(\mathrm{W} \mathrm{m}^{-1} \mathrm{~K}^{-1}\right)$.

$\phi$, incoming heat flux on a particle $\left(\mathrm{W} \mathrm{m}^{-2}\right)$.

$\rho$, density $\left(\mathrm{kg} \mathrm{m}^{-3}\right)$.

$\sigma$, specific area ratio $\left(\mathrm{m}^{-1}\right)$.

$\theta$, dimensionless temperature (unitless).

$\theta_{0}$, steady-state solution (unitless).

$\theta_{1}$, time-dependent perturbation (unitless).

\section{Subscripts}

char, charring.

conv, convective.

ig, ignition.

$l$, liquid.

pyr, pyrolysis.

$\mathrm{rad}$, radiative.

$s$, solid.

sim, simulation.

vap, vapour.

$w$, wood.

$x p$, experiment.

$\infty$, reference (surrounding air).

\section{Dimensionless numbers}

$\mathrm{Bi}$, Biot number.

$F M C$, fuel moisture content.

$F M C_{v}$, volumetric fuel moisture content.

$\mathrm{Gr}$, Grashof number.

$\mathrm{Nu}$, Nusselt number.

Pr, Prandtl number.

$\Phi$, dimensionless incoming heat flux on a particle.

\section{Acknowledgements}

The authors thank Jan C. Thomas from University of Edinburgh for giving access to the original data from his Ph.D. thesis for the $110 \%$ FMC case. Authors also thank the Associate Editor and the reviewers who helped making this article clearer. This work was granted access to the HPC resources of Aix-Marseille Université financed by the project Equip@Meso (ANR-10-EQPX-29-01) of the program 'Investissements d'Avenir' supervised by the Agence Nationale pour la Recherche.

\section{References}

Anand C, Shotorban B, Mahalingam S, McAllister S, Weise DR (2017) Physics-based modeling of live wildland fuel ignition experiments in the forced ignition and flame spread test apparatus. Combustion Science and Technology 189, 1551-1570. doi:10.1080/00102202.2017.1308357

Chaos M (2014) Spectral aspects of bench-scale flammability testing: application to hardwood pyrolysis. Fire Safety. Science 11, 165-178. doi:10.3801/IAFSS.FSS.11-165

Di Blasi C, Branca C, Santoro A, Hernandez EG (2001) Pyrolytic behaviour and products of some wood varieties. Combustion and Flame 124, 165-177. doi:10.1016/S0010-2180(00)00191-7

El Houssami M (2016) Development of a numerical and experimental framework to understand and predict the burning dynamics of porous fuel beds. PhD thesis, The University of Edinburgh. Available at https:// www.era.lib.ed.ac.uk/handle/1842/23465 [Verified 30 January 2017]

El Houssami M, Thomas JC, Lamorlette A, Morvan D, Chaos M, Hadden R, Simeoni A (2016) Experimental and numerical studies characterizing the burning dynamics of wildland fuels. Combustion and Flame 168, 113-126. doi:10.1016/J.COMBUSTFLAME.2016.04.004

Grishin AM (1997) 'A mathematical modelling of forest fires and new methods of fighting them.' (Publishing House of the Tomsk University: Tomsk, Russia)

Irvine TF, Hartnett JP (1978) 'Advances in heat transfer', vol. 4. (Academic Press: New York, NY, USA)

Lamorlette A (2014) Quantification of ignition time uncertainty based on the classical ignition theory and fourier analysis. Comptes Rendus. Mécanique 342, 459-465. doi:10.1016/J.CRME.2014.06.002

Lamorlette A, Candelier F (2015) Thermal behavior of solid particles at ignition: theoretical limit between thermally thick and thin solids. International Journal of Heat and Mass Transfer 82, 117-122. doi:10.1016/J.IJHEATMASSTRANSFER.2014.11.037

Larini M, Giroud F, Porterie B, Louraud JC (1998) A multiphase formulation for fire propagation in heterogeneous combustible media. International Journal of Heat and Mass Transfer 41, 881-897. doi:10.1016/ S0017-9310(97)00173-7

Liukov AV (1968) 'Analytical heat diffusion theory.' (Academic Press: New York, NY, USA)

McAllister S, Grenfell I, Hadlow A, Jolly WM, Finney M, Cohen J (2012) Piloted ignition of living forest fuels. Fire Safety Journal 51, 133-142. doi:10.1016/J.FIRESAF.2012.04.001

Mell W, Maranghides A, McDermott R, Manzelle SL (2009) Numerical simulation and experiments of burning Douglas-fir trees. Combustion and Flame 156, 2023-2041. doi:10.1016/J.COMBUSTFLAME.2009. 06.015

Morvan D, Dupuy JL (2001) Modelling of fire spread through a forest fuel bed using a multiphase formulation. Combustion and Flame 127, 19811994. doi:10.1016/S0010-2180(01)00302-9

Morvan D, Méradji S, Accary G (2009) Physical modelling of fire spread in grasslands. Fire Safety Journal 44, 50-61. doi:10.1016/J.FIRESAF. 2008.03.004

Nepf HM (1999) Drag, turbulence, and diffusion in flow through emergent vegetation. Water Resources Research 35, 479-489. doi:10.1029/ 1998WR900069

Patankar S (1980) 'Numerical heat transfer and fluid flow.' (McGraw-Hill: New York, NY, USA)

Pickett BM, Isackson C, Wunder R, Fletcher TH, Butler BW, Weise DR (2010) Experimental measurements during combustion of moist individual foliage samples. International Journal of Wildland Fire 19, 153-162. doi:10.1071/WF07121 
Ren N, Wang Y, Vilfayeau S, Trouvé A (2016) Large eddy simulation of turbulent vertical wall fires supplied with gaseous fuel through porous burners. Combustion and Flame 169, 194-208. doi:10.1016/J.COM BUSTFLAME.2015.12.008

Schemel CF, Simeoni A, Biteau H, Rivera JD, Torero JL (2008) A calorimetric study of wildland fuels. Experimental Thermal and Fluid Science 32, 1381-1389. doi:10.1016/J.EXPTHERMFLUSCI.2007. 11.011

Séro-Guillaume O, Margerit J (2002) Modelling forest fires. Part I: a complete set of equations derived by extended irreversible thermodynamics. International Journal of Heat and Mass Transfer 45, 1705 1722. doi:10.1016/S0017-9310(01)00248-4

Shaw RH, Patton EG (2003) Canopy element influences on resolved- and sub-grid-scale energy within a large-eddy simulation. Agricultural and Forest Meteorology 115, 5-17. doi:10.1016/S0168-1923(02)00165-X
Simpson W, TenWolde A (1999) Wood handbook: wood as an engineering material. USDA Forest Service, Forest Products Laboratory. General technical report FPL; GTR-113. (Madison, WI, USA)

Thomas JC (2016) Improving the understanding of fundamental mechanisms that influence ignition and burning behavior of porous wildland fuel beds. $\mathrm{PhD}$ thesis, The University of Edinburgh.

Torero JL (2008) 'SFPE handbook of fire protection engineering', 5th edn. pp. 633-662. (National Fire Protection Association: New York, NY, USA)

Torero JL, Simeoni A (2010) Heat and mass transfer in fires: scaling laws, ignition of solid fuels and application to forest fires. The Open Thermodynamics Journal 4, 145-155. doi:10.2174/1874396X01004010145 